\title{
Long-read sequencing of 12 samples discovered novel variants within human leukocyte antigen region
}

\author{
Sung Min Kim, Young Jin Kim
}

Division of Genome Science, Department of Precision Medicine, National Institute of Health, Cheongju, Korea

Background: The human leukocyte antigen (HLA) region is known as the most polymorphic region in the human. In clinical transplantation, increased HLA mismatch between donor and recipient were a well-known risk factor for graft survival. Despite its importance, variant discovery within HLA is not feasible due to its extreme genetic diversity and highly similar sequences. Recently, PacBio Sequel II provides high-fidelity (HiFi) long-read data of $\sim 15 \mathrm{~kb}$ enabling us to accurately resolve highly complex genomic regions. Here, we used PacBio HiFi long-read sequencing to obtain novel HLA variants.

Methods: Five megabytes of HLA regions were targeted and 12 samples were sequenced using PacBio HiFi sequencing technology. Sequenced long-reads were mapped to the hg19 reference genome using minimap2. Variants within HLA regions were called using DeepVariant and GLnexus. Discovered HLA region variants were compared to those of gnomAD database and Han Chinese $\mathrm{MHC}$ reference panel (HanREF, $n=10,689)$.

Results: Mean read length and read depth of HiFi sequencing were $4.8 \mathrm{~kb}$ and $77.7 \mathrm{x}$, respectively. These results implied enhanced information compared to those of short-read sequencing data (e.g., HanREF with $\sim 55 \mathrm{x}$ and 90 bp paired-end reads). In overall, 69,318 variants were discovered from 12 samples. Among them, about 50,000 variants were previously catalogued in gnomAD. However, long-read sequencing data produced three times more variants than those of HanREF ( 18,000 overlapped variants).

Conclusions: Long-read sequencing provided numerous novel HLA variants. The discovered variants will be a valuable resource for constructing HLA reference panel and identifying novel HLA alleles. Since an accuracy of HiFi sequencing is relatively low compared to established genomic platforms, however, the novel variants should be validated using other genomic platforms.

Corresponding author: Young Jin Kim

E-mail: anwltlarkr@gmail.com

(c) The Korean Society for Transplantation

This is an Open Access article distributed under the terms of the Creative Commons Attribution Non-Commercial License (http://creativecommons.org/licenses/by-nc/4.0/) which permits unrestricted non-commercial use, distribution, and reproduction in any medium, provided the original work is properly cited 